\title{
Mechanism of interactions between endoplasmic reticulum stress and autophagy in hypoxia/reoxygenation-induced injury of $\mathrm{H9c2}$ cardiomyocytes
}

\author{
GAOPENG GUAN $^{1-3^{*}}$, LEI YANG ${ }^{2,4^{*}}$, WENYIN HUANG ${ }^{1}$, JUN ZHANG $^{1}$, \\ PUHUA ZHANG ${ }^{1}$, HUAN YU ${ }^{2,4}$, SHENGYUAN LIU ${ }^{1,2}$ and XIANG GU ${ }^{1,2}$ \\ ${ }^{1}$ Department of Cardiology, Affiliated Hospital of Jiujiang University; \\ ${ }^{2}$ Key Laboratory of System Bio-Medicine of Jiangxi Province, Jiujiang University, Jiujiang, Jiangxi 332000; \\ ${ }^{3}$ Department of Cardiology, The First Affiliated Hospital of Nanchang University, Nanchang, Jiangxi 330006; \\ ${ }^{4}$ Basic Medical Science College, Jiujiang University, Jiujiang, Jiangxi 332000, P.R. China
}

Received October 10, 2018; Accepted April 25, 2019

DOI: $10.3892 / \mathrm{mmr} .2019 .10228$

\begin{abstract}
Endoplasmic reticulum (ER) stress and autophagy are involved in myocardial ischemia-reperfusion (I/R) injury; however, their roles in this type of injury remain unclear. The present study investigated the roles of ER stress and autophagy, and their underlying mechanisms, in H9c2 cells during hypoxia/reoxygenation (H/R) injury. Cell viability was detected by CCK-8 assay. The autophagy flux was monitored with mCherry-GFP-LC3-adenovirus transfection. The expression levels of autophagy-related proteins and ER stress-related proteins were measured by western blotting. Apoptosis was detected by flow cytometry and western blotting. The results indicated that autophagy was induced, ER stress was activated and apoptosis was promoted in H9c2 cells during H/R injury. The inhibition of ER stress by 4-phenylbutyrate or C/EBP homologous protein (CHOP)-targeting small interfering RNA (siRNA) decreased autophagy and ameliorated cell apoptosis during $\mathrm{H} / \mathrm{R}$ injury. Activation of autophagy by rapamycin attenuated ER stress and ameliorated cell apoptosis. Inhibition of autophagy by 3-methyladenine or Beclin1-targeting siRNA aggravated ER stress and exacerbated cell apoptosis, and activation of ER stress by thapsigargin decreased autophagy and induced cell apoptosis. Collectively, the findings of the present study demonstrated that $\mathrm{H} / \mathrm{R}$ induced apoptosis and autophagy via ER stress in H9c2 cells, and that CHOP may serve an important role in ER stress-induced autophagy and
\end{abstract}

Correspondence to: Dr Xiang Gu, Department of Cardiology, Affiliated Hospital of Jiujiang University, 17 Lufeng Road, Jiujiang, Jiangxi 332000, P.R. China

E-mail: eagle0094@163.com

*Contributed equally

Key words: hypoxia/reoxygenation injury, endoplasmic reticulum stress, autophagy, apoptosis, H9c2 apoptosis. Autophagy, as an adaptive response, was activated by ER stress and alleviated ER stress-induced cell apoptosis during $H / R$ injury.

\section{Introduction}

Acute myocardial infarction (AMI) is a serious cardiovascular disease caused by coronary occlusion, leading to acute and persistent ischemia and hypoxia in the myocardium. Revascularization by thrombolysis or percutaneous coronary intervention (PCI) is the main treatment for AMI, and effectively reduces AMI injury and limits the size of the myocardial infarction (1); however, myocardial reperfusion injury may be induced by the reestablished blood flow in the ischemic myocardium (2). Therefore, ischemia/reperfusion (I/R) injury is a major cause of AMI damage. Hypoxia/reoxygenation $(\mathrm{H} / \mathrm{R})$ is an important factor in the pathogenesis of tissue I/R injury (3).

Previous studies have reported that endoplasmic reticulum (ER) stress and autophagy are associated with I/R $(4,5)$. The ER is an indispensable eukaryotic organelle that is primarily responsible for the translocation and post-transcriptional modification of proteins, and the maintenance of cellular calcium $\left(\mathrm{Ca}^{2+}\right)$ homeostasis and lipid synthesis (6); however, under extreme conditions, such as $\mathrm{I} / \mathrm{R}$, the homeostasis is disrupted, inducing ER stress, which leads to the apoptosis of cells (7). Autophagy is a highly conserved catabolic process involving the degradation and recycling of excess/impaired proteins and organelles via autophagosome formation and lysosome degradation, serving an important role in maintaining cell homeostasis (8); however, under stressful conditions, such as hypoxia or starvation, autophagy is activated as a pathway to promote cell survival by conserving energy and reducing toxic substances (9).

A previous study reported that the activation of ER stress induces cardiomyocyte apoptosis during H/R (10), and a separate study reported that autophagy serves a protective role against $\mathrm{H} / \mathrm{R}$ injury (11). In addition, an association between ER stress and autophagy was reported in ovarian injury and HCT116 cell apoptosis $(12,13)$; however, whether there is potential crosstalk 
between autophagy and ER stress during cardiomyocyte $\mathrm{H} / \mathrm{R}$ injury is yet to be determined. Thus, the present study aimed to investigate the roles and mechanisms of ER stress and autophagy in $\mathrm{H} 9 \mathrm{c} 2$ cell apoptosis during $\mathrm{H} / \mathrm{R}$ injury.

\section{Materials and methods}

Materials. Rapamycin (Rap, autophagy agonist), 3-methyladenine (3-MA, autophagy inhibitor), thapsigargin (Tg, ER stress agonist) and 4-phenylbutyrate (4-PBA, ER stress inhibitor) were purchased from Sigma-Aldrich (Merck KGaA). Fetal bovine serum (FBS) was purchased from Gibco (Thermo Fisher Scientific, Inc.). High-glucose Dulbecco's Modified Eagle's medium (DMEM) was obtained from HyClone (GE Healthcare Life Sciences). The rat H9c2 cell line was purchased from the Chinese Academy of Sciences. Caspase-3 Activity Assay kit (cat. no. G015-1-3), Lactate Dehydrogenase (LDH) Assay kit (cat. no. A020-2-2), radio immunoprecipitation assay (RIPA) lysis buffer and Bicinchoninic Acid (BCA) Protein Assay kits were purchased from Nanjing Jiancheng Bio-Engineering Institute Co., Ltd. A Cell Counting Kit-8 (CCK-8) and an Annexin V-fluorescein isothiocyante (FITC)/propidium iodide (PI) Apoptosis Analysis kit were purchased from Beijing Zoman Biotechnology Co., Ltd. TRIzol ${ }^{\circledR}$ Reagent was purchased from Invitrogen (Thermo Fisher Scientific, Inc.) and a First Strand cDNA Synthesis kit was purchased from Tiangen Biotech Co., Ltd. A SYBR Green Master Mix kit was purchased from Takara Bio, Inc. Adenovirus expressing mCherry-green fluorescent protein (GFP)-microtubule-associated proteins $1 \mathrm{~A} / 1 \mathrm{~B}$ light chain 3B (LC3B) was obtained from Beyotime Institute of Biotechnology. Control small interfering RNA (siRNA), specific siRNA for Beclin1 and specific siRNA for C/EBP homologous protein (CHOP) were obtained from Shanghai GeneChem Co., Ltd. Lipofectamine ${ }^{\circledR} 2000$ was obtained from Invitrogen (Thermo Fisher Scientific, Inc.). The primary antibodies rabbit anti-BAX (cat. no. sc-6236), rabbit anti-Bcl2 (cat. no. sc-23960) and rabbit anti-GAPDH (cat. no. sc-32233) were obtained from Santa Cruz Biotechnology, Inc. The primary antibodies rabbit anti-activating transcription factor 6 (ATF6; cat. no. ab37149), rabbit anti-CHOP (cat. no. ab10444), rabbit anti-glucose-regulated protein 78 (GRP78; cat. no. ab32618), rabbit anti-Beclin1 (cat. no. ab62557), rabbit anti-P62 (cat. no. ab91526) and rabbit anti-LC3 (cat. no. ab48394) were purchased from Abcam. The goat anti-rabbit secondary antibodies (cat. no. SA00001-2) were obtained from ProteinTech Group, Inc.

Cell culture and treatment. H9c2 cells were cultured in DMEM containing $10 \% \mathrm{FBS}, 50 \mathrm{U} / \mathrm{ml}$ penicillin and $50 \mu \mathrm{g} / \mathrm{ml}$ streptomycin, and incubated at $37^{\circ} \mathrm{C}$ in a humidified atmosphere containing $5 \% \mathrm{CO}_{2} / 95 \%$ air. For hypoxia treatment, the $\mathrm{H} 9 \mathrm{c} 2$ cell media were replaced with serum-free and glucose-free DMEM prior to incubation in an anaerobic chamber with a humidified atmosphere consisting of $5 \% \mathrm{CO}_{2} / 95 \% \mathrm{~N}_{2}$ for 3 , 6,12 and $18 \mathrm{~h}$. The cells were then subjected to reperfusion by replacing the media with high-glucose DMEM containing $10 \%$ FBS followed by incubation under normoxic conditions for $6 \mathrm{~h}$ and 12/6 h H/R was used for all $\mathrm{H} / \mathrm{R}$ treatment following the initial viability, $\mathrm{LDH}$, and apoptosis experiments. In the experimental group, the cells were pretreated with Rap $(5 \mu \mathrm{M})$, 3 -MA $(5 \mathrm{mM})$ or 4 -PBA $(5 \mu \mathrm{M})$ at $37^{\circ} \mathrm{C}$ for $4 \mathrm{~h}$ prior to $\mathrm{H} / \mathrm{R}$, and the positive control cells were treated with Rap $(5 \mu \mathrm{M})$, 3 -MA $(5 \mathrm{mM}), 4-\mathrm{PBA}(5 \mu \mathrm{M})$ or $\mathrm{Tg}(2 \mu \mathrm{M})$ in normoxic conditions at $37^{\circ} \mathrm{C}$ for $4 \mathrm{~h}$ before the cells were cultured in normal medium for $14 \mathrm{~h}$.

Cell viability and lactate dehydrogenase $(L D H)$ assays. A CCK-8 assay was performed to determine cell viability. Briefly, cells were seeded at a density of $1 \times 10^{4}$ cells/well in 96-well plates. Following the induction of $\mathrm{H} / \mathrm{R}$, cells were incubated with $10 \mu \mathrm{l}$ CCK-8 solution for an additional $2 \mathrm{~h}$ at $37^{\circ} \mathrm{C}$, and the absorbance value was measured at a wavelength of $450 \mathrm{~nm}$ using a microplate reader. Cell injury was verified using an LDH assay. After treatment, $0.2 \mathrm{ml}$ culture medium was used to measure LDH activity using an LDH assay kit according to the manufacturer's protocol. The CCK-8 and $\mathrm{LDH}$ results were presented as a percentage of the values measured for control cells that were incubated under normoxic conditions for the same time.

Measurement of apoptosis by flow cytometry. The apoptotic rates of $\mathrm{H} 9 \mathrm{c} 2$ cells were determined using an Annexin V-FITC Apoptosis Detection kit. Briefly, $1 \times 10^{5} / \mathrm{ml} \mathrm{H9c2} \mathrm{cells} \mathrm{were}$ inoculated into 6 -well culture plates at $37^{\circ} \mathrm{C}$ for $24 \mathrm{~h}$. Following the different treatments, the cells were collected and resuspended in $500 \mu \mathrm{l}$ binding buffer. After incubation with $10 \mu \mathrm{l}$ Annexin V-FITC and $5 \mu \mathrm{l}$ PI for $15 \mathrm{~min}$ at room temperature in the dark, the apoptotic rates of the cells were measured using a flow cytometer (FACSCalibur ${ }^{\mathrm{TM}}$; BD Biosciences) and analyzed using CellQuest Pro software (version 3.3, BD Biosciences).

siRNA transfection. The siRNA sequences used were as follows: Control, 5'-UUCUCCGAACGUGUCACGUTT-3'; Beclin1, 5'-GAUGGUGUCUCUCGAAGAUdTdT-3'; CHOP, 5'-GGUCCUGUCCUCAGAUGAAdTdT-3'. 1×105 cells $/ \mathrm{ml}$ $\mathrm{H} 9 \mathrm{c} 2$ cells were seeded into 6 -well plates and cultured to $70-80 \%$ confluence. $\mathrm{H} 9 \mathrm{c} 2$ cells were transfected with $10 \mathrm{nM}$ control siRNA (Con siRNA), Beclin1 siRNA or CHOP siRNA using Lipofectamine ${ }^{\circledR} 2000$ according to the manufacturer's protocols. H/R or other treatments were performed at $6 \mathrm{~h}$ post-transfection.

Reverse transcription-quantitative polymerase chain reaction $(R T-q P C R)$. Total RNA was isolated from H9c2 cells using TRIzol ${ }^{\circledR}$ Reagent. The total RNA of each sample was quantified using a spectrophotometer (NanoDrop Technologies; Thermo Fisher Scientific, Inc.). RNA (1 $\mu \mathrm{g})$ was reverse-transcribed in a $20 \mu \mathrm{l}$ reaction volume with oligo dT primers using a First Strand cDNA Synthesis kit (Tiangen Biotech Co., Ltd). qPCR was performed using an Applied Biosystems 7500 Real-Time PCR system (Applied Biosystems; Thermo Fisher Scientific, Inc.) with a SYBR Green Master Mix kit. The PCR cycling conditions consisted of initial denaturation at $95^{\circ} \mathrm{C}$ for $1 \mathrm{~min}$, followed by 40 cycles at $95^{\circ} \mathrm{C}$ for $15 \mathrm{sec}$ and at $60^{\circ} \mathrm{C}$ for $34 \mathrm{sec}$. The expression levels of Beclin1 and CHOP were normalized to GAPDH. Relative quantification of gene expression was performed using the $2^{-\Delta \Delta \mathrm{Cq}}$ method (14). The mRNA primers used were: Beclin1, forward 5'-GCCTCTGAAACT GGACACG-3', reverse, 5'-CCTCTTCCTCCTGGCTCTCT-3'; CHOP, forward 5'-CTGGAAGCCTGGTATGAGGAT-3', 

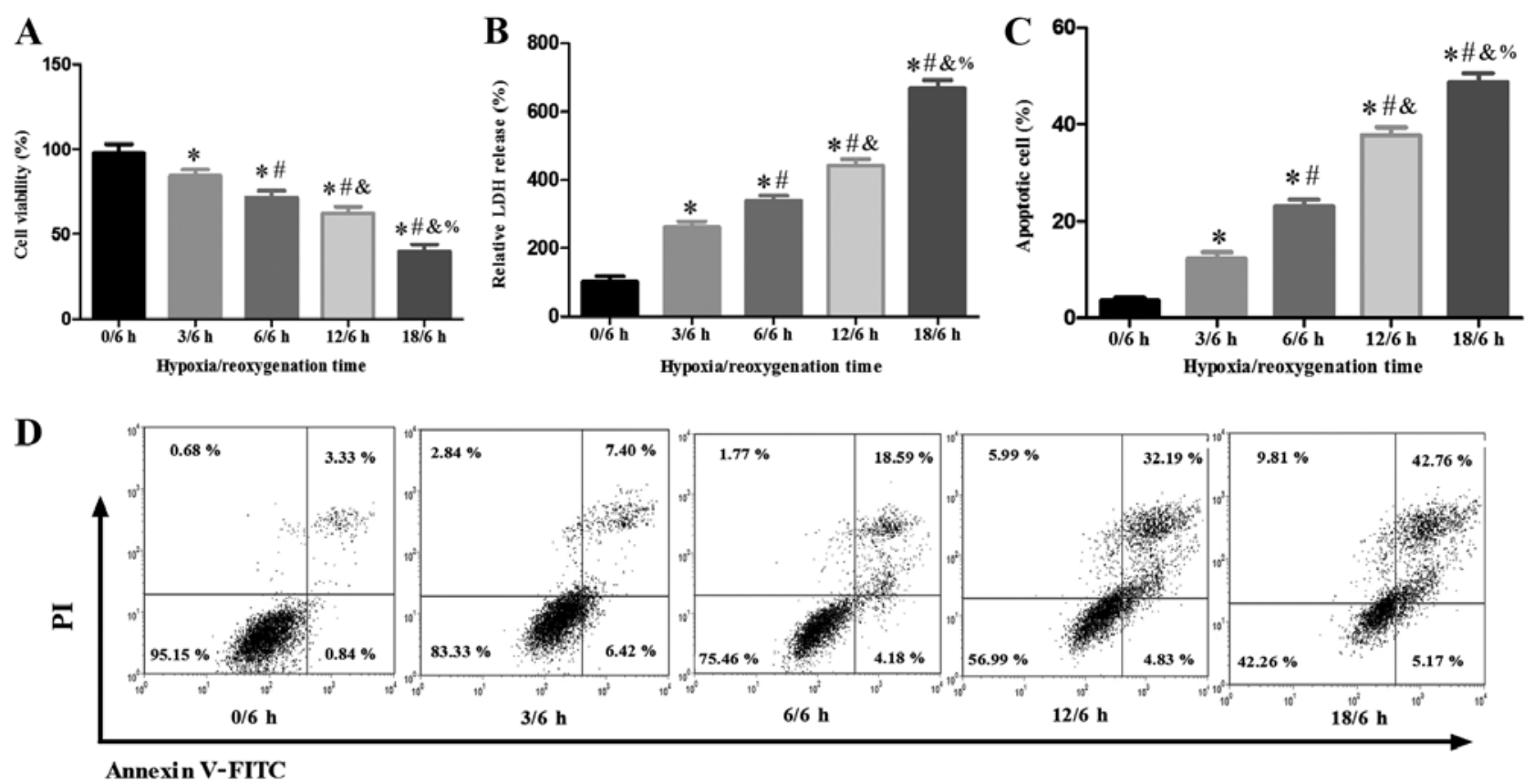

Figure 1. Determination of the cytotoxicity of H/R to H9c2 cells at various time intervals. Cells were subjected to hypoxia of varying duration followed by reoxygenation for $6 \mathrm{~h}$, and then the (A) viability and (B) LDH release of cells were determined via Cell Counting Kit-8 and LDH assays. (C and D) Apoptosis of $\mathrm{H} / \mathrm{R}$-treated $\mathrm{H} 9 \mathrm{c} 2$ cells following various periods of hypoxia. Data are presented as the means \pm standard error of the mean of three independent experiments. ${ }^{*} \mathrm{P}<0.05$ vs. $0 / 6 \mathrm{~h} ;{ }^{~} \mathrm{P}<0.05$ vs. $3 / 6 \mathrm{~h} ;{ }^{\&} \mathrm{P}<0.05$ vs. $6 / 6 \mathrm{~h} ;{ }^{\%} \mathrm{P}<0.05$ vs. $12 / 6 \mathrm{~h} . \mathrm{H} / \mathrm{R}$, hypoxia/reoxygenation; $\mathrm{LDH}$, lactate dehydrogenase; PI, propidium iodide.

reverse, 5'-CAGGGTCAAGAGTAGTGAAGGT-3'; and GAPDH, forward 5'-CTCGTCTCATAGACAAGATGGT-3' and reverse, 5'-GGGTAGAGTCATACTGGAACATG-3'.

Western blotting. After treatment, the cells were collected, washed with ice-cold PBS and lysed with RIPA buffer; the total protein concentration was determined via a BCA assay. Subsequently, $30 \mu \mathrm{g} /$ lane total protein was separated via $15 \%$ SDS-PAGE (with the exception of experiments involving CHOP siRNA, for which the concentration of total protein used was $50 \mu \mathrm{g}$ ), after which they were transferred to PVDF membranes. After blocking in TBS-0.1\% Tween-20 with $10 \%$ non-fat milk for $2 \mathrm{~h}$ at room temperature, the samples were incubated overnight at $4{ }^{\circ} \mathrm{C}$ with primary antibodies against $\mathrm{Bcl} 2$ (1:1,000), ATF6 (1:1,000), BAX (1:500), GAPDH $(1: 2,000)$, CHOP $(1: 1,000)$, GRP78 $(1: 1,000)$ Beclin1 (1:1,000), P62 (1:1,000) and LC3 (1:1,000). After washing, the membranes were incubated with secondary antibody $(1: 4,000)$ conjugated to horseradish peroxidase at $37^{\circ} \mathrm{C}$ for $30 \mathrm{~min}$. The immunoreactive bands were visualized using a Super Signal West Pico kit (Beijing Solarbio Science \& Technology Co., Ltd.) according to the manufacturer's protocol, and the protein band densities were semi-quantified by densitometric analysis using ImageJ software (version 1.48, National Institutes of Health).

mCherry-GFP-LC3 adenovirus transduction and autophagy assay. $\mathrm{H} 9 \mathrm{c} 2$ cells cultured in $24-w e l l$ plates $\left(1 \times 10^{5}\right.$ cells/well $)$ were transduced with mCherry-GFP-LC3 adenovirus at 40 MOI (multiplicity of infection) for $24 \mathrm{~h}$ at $37^{\circ} \mathrm{C}$ in a humidified atmosphere containing $5 \% \mathrm{CO}_{2} / 95 \%$ air. Following transduction, the cells were incubated with fresh culture medium for $24 \mathrm{~h}$ at $37^{\circ} \mathrm{C}$. The numbers of GFP and mCherry dots per cell were counted in three randomly selected fields under a fluorescence microscope (Olympus Corporation).

Caspase-3 activity measurement. Caspase-3 activity was measured using a Caspase-3 Activity Colorimetric Assay kit. Briefly, following the various aforementioned treatments, cells were harvested by scraping, collected by centrifugation at $800 \times \mathrm{g}$ and $4^{\circ} \mathrm{C}$ for $5 \mathrm{~min}$, and lysed with RIPA buffer on ice for $15 \mathrm{~min}$. Subsequently, the lysate was centrifuged at $12,000 \mathrm{x} \mathrm{g}$ and $4^{\circ} \mathrm{C}$ for $15 \mathrm{~min}$, and the protein content was determined, following which the caspase-3 substrate was measured at $405 \mathrm{~nm}$ using a microplate reader (Model 680, Bio-Rad Laboratories, Inc.).

Statistical analyses. All experiments were repeated at least three times for each group, and the data are presented as the means \pm standard error of the mean. The data were analyzed by one-way analysis of variance followed by Fisher's least significant difference test using SPSS version 13.0 software (SPSS, Inc.).

\section{Results}

Effects of $H / R$ treatment on damage and apoptosis in $H 9 c 2$ cells. To investigate the injurious effects of $\mathrm{H} / \mathrm{R}$ on $\mathrm{H} 9 \mathrm{c} 2$ cells, cells were subjected to hypoxia for $0,3,6,12$ and $18 \mathrm{~h}$ prior to reperfusion for $6 \mathrm{~h}$. H9c2 cell viability was significantly decreased in a time-dependent manner under hypoxic conditions for $0,3,6,12$ and $18 \mathrm{~h}$ followed by reperfusion for $6 \mathrm{~h}$, with a $\sim 40 \%$ reduction in viability following $12 \mathrm{~h}$ of hypoxia (Fig. 1A). LDH results indicated that H/R induced a significant increase in the release of $\mathrm{LDH}$ in $\mathrm{H} 9 \mathrm{c} 2$ cells in a time-dependent manner (Fig. 1B). Consistent with these 
$\mathbf{A}$

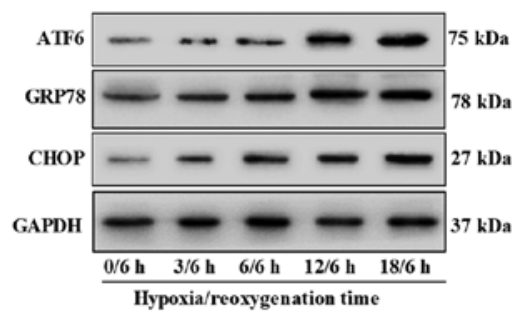

B

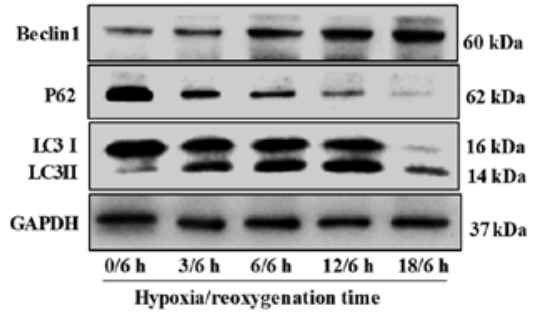

C

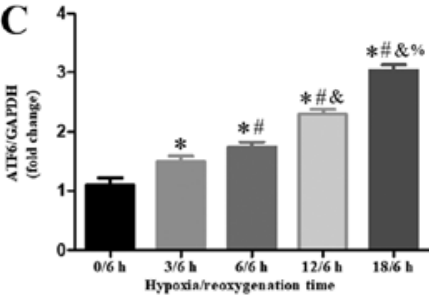

$\mathbf{E}$
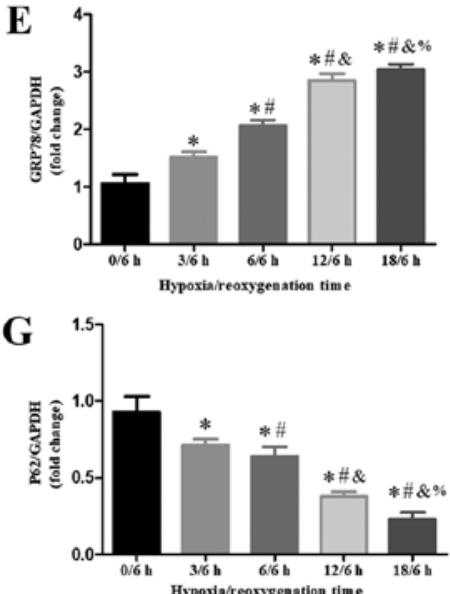

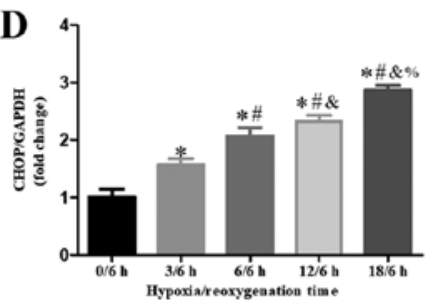

$\mathbf{F}$
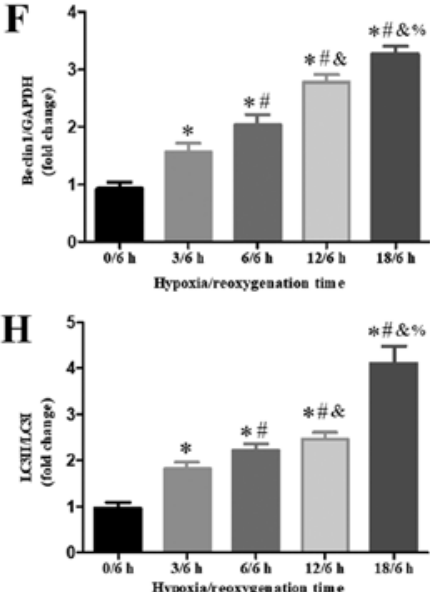

Figure 2. Endoplasmic reticulum stress and autophagy in H9c2 cells following H/R. (A) Expression of ATF6, GRP78 and CHOP following various periods of hypoxia and $6 \mathrm{~h}$ of reoxygenation in H9c2 cells. (B) Expression of Beclin1, P62, and LC3 following various periods of hypoxia and $6 \mathrm{~h}$ of reoxygenation in H9c2 cells. (C-H) Semi-quantification of the relative ratio of ATF6, GRP78, CHOP, Beclin1, P62 and LC3II/LC3I following H/R; protein expression levels were normalized to GAPDH. Data are presented as the means \pm standard error of the mean of three independent experiments. ${ }^{*} \mathrm{P}<0.05$ vs. $0 / 6 \mathrm{~h} ;{ }^{\#} \mathrm{P}<0.05$ vs. $3 / 6 \mathrm{~h} ;{ }^{\&} \mathrm{P}<0.05$ vs. $6 / 6 \mathrm{~h} ;{ }^{\%} \mathrm{P}<0.05$ vs. $12 / 6 \mathrm{~h}$. ATF6, activating transcription factor 6 ; CHOP, C/EBP homologous protein; GRP78, glucose-regulated protein 78; $\mathrm{H} / \mathrm{R}$, hypoxia/reoxygenation; LC3, microtubule-associated proteins $1 \mathrm{~A} / 1 \mathrm{~B}$ light chain $3 \mathrm{~B}$.

findings, as the duration of $\mathrm{H} / \mathrm{R}$ increased, the number of apoptotic H9c2 cells increased (Fig. 1C and D). It was revealed that $\mathrm{H} / \mathrm{R}$ treatment of $\mathrm{H} 9 \mathrm{c} 2$ cells for $12 / 6 \mathrm{~h}$ resulted in the apoptosis of $>30 \%$ of cells. Therefore, $12 / 6 \mathrm{~h} \mathrm{H} / \mathrm{R}$ was used for all $\mathrm{H} / \mathrm{R}$ treatments following the viability, $\mathrm{LDH}$ and apoptosis experiments.

Effects of H/R-treatment on autophagy and ER stress in $H 9 c 2$ cells. Western blot analysis suggested that the expression patterns of Beclin1 and LC3-II/LC3-I were similar. Beclin1 expression and LC3II/LC3I ratio were increased in a time-dependent manner following $\mathrm{H} / \mathrm{R}$, whereas the expression of P62 protein was downregulated (Fig. 2B and F-H). These results indicated that the expression of Beclin1 and ratio of LC3II/LC3I were promoted but the expression of P62 was inhibited by H/R. In addition, fluorescent microscopy revealed that $\mathrm{H} / \mathrm{R}$ promoted the formation of autophagosomes and autolysosomes compared with the control (Fig. 3C and D). These results indicated that autophagy was activated in H/R-treated H9c2 cells. Additionally, the expression levels of ER stress proteins (ATF6, GRP78 and CHOP) were upregulated following $\mathrm{H} / \mathrm{R}$ in a time-dependent manner (Fig. 2A and C-E).

Autophagy is altered by ER stress levels in H/R-treated $H 9 c 2$ cells. To determine whether autophagy was influenced by ER stress in cells during H/R injury, 4-PBA was used to inhibit ER stress during treatment (Fig. 3A). 4-PBA pretreatment significantly decreased the expression levels of ATF6, GRP78 and CHOP compared with in the H/R treatment group. Additionally, 4-PBA pretreatment effectively decreased Beclin1 expression and LC3-II/LC3-I ratio, but increased P62 expression, compared with the $\mathrm{H} / \mathrm{R}$ treatment group, and decreased the formation of H/R-induced autophagosomes and autolysosomes (Fig. 3C and D). Conversely, Tg (an ER stress agonist) significantly increased the expression levels of ATF6, GRP78 and CHOP in H9c2 cells, and the expression of the autophagy-associated proteins Beclin1 and LC3, but not P62, was increased by Tg compared with control group in H9c2 cells (Fig. 3A). The numbers of autophagosomes and autolysosomes were also significantly increased following the treatment of $\mathrm{H} 9 \mathrm{c} 2$ cells with $\mathrm{Tg}$ (Fig. 3C and D).

ER stress is altered by autophagy activity in H/R-treated $H 9 c 2$ cells. To determine whether ER stress was influenced by autophagy activity in HR injury, Rap or 3-MA were used to activate or inhibit autophagy prior to treatment, respectively (Fig. 3B). The results revealed that Rap pretreatment effectively increased Beclin1 expression and LC3-II/LC3-I ratio, but decreased P62 expression, during H/R treatment, and in the absence of H/R treatment. Conversely, 3-MA pretreatment successfully decreased Beclin1 expression and LC3-II/LC3-I ratio, but increased $\mathrm{P} 62$ expression, in $\mathrm{H} 9 \mathrm{c} 2$ cells during $\mathrm{H} / \mathrm{R}$ treatment. Additionally, Rap pretreatment increased the formation of H/R-induced autophagosomes and autolysosomes, whereas 3-MA pretreatment induced opposing effects (Fig. 3C and D). Rap pretreatment also resulted in a significant decrease in the protein expression of ATF6, GRP78 and CHOP during H/R, whereas 3-MA pretreatment significantly increased their protein expression (Fig. 3B). 
A

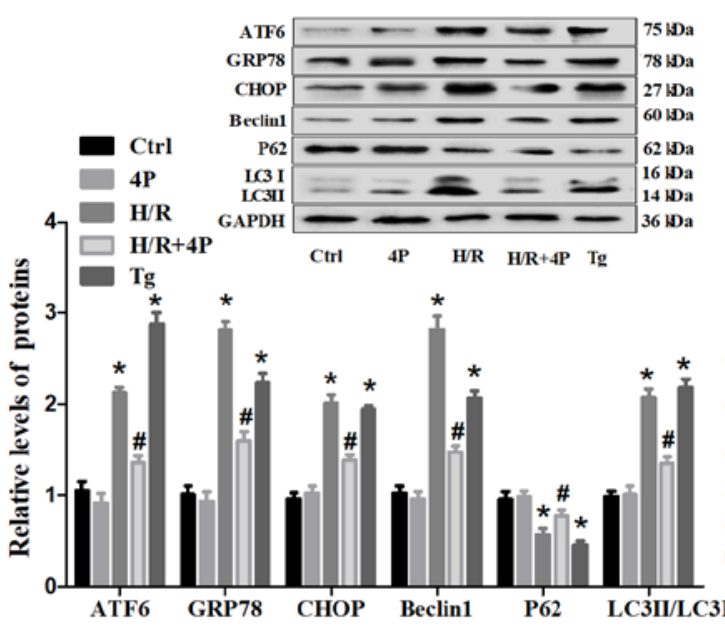

B

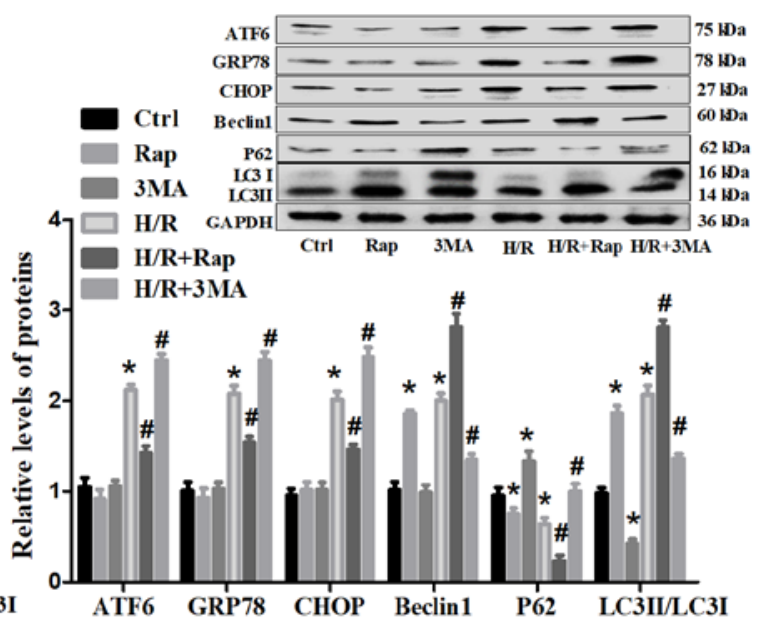

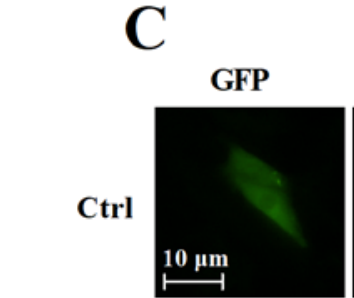
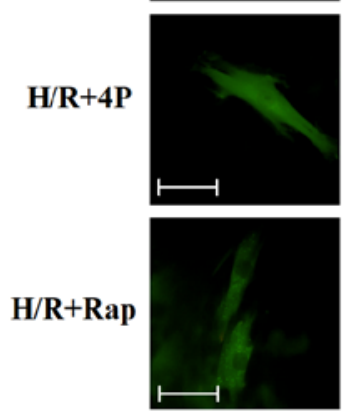

mCherry
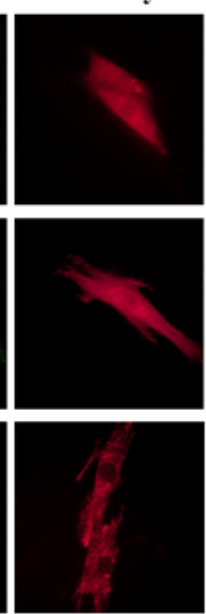

$\mathbf{H} / \mathbf{R}$
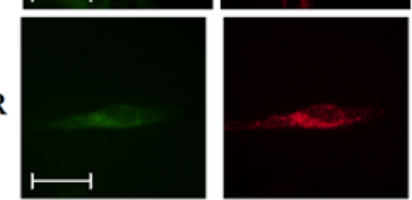
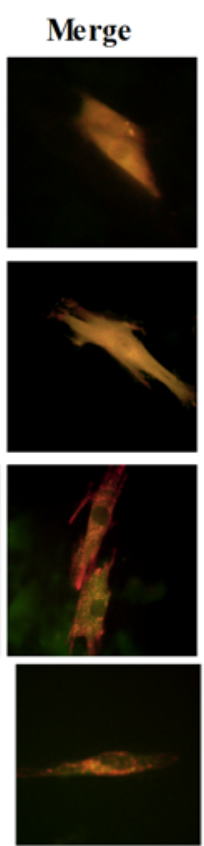
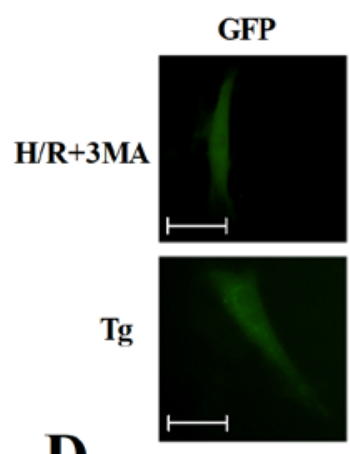

D
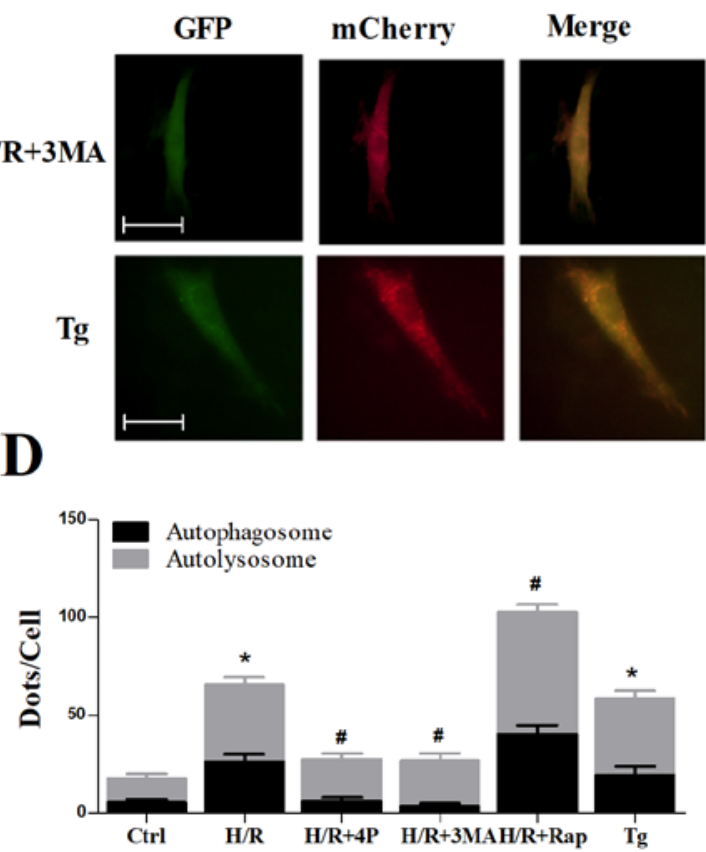

Figure 3. Effects of Rap, 3-MA, Tg and 4-PBA on the autophagy and endoplasmic reticulum stress of H/R-treated H9c2 cells. Untreated H9c2 cells, or cells pretreated with Rap, 3-MA or 4-PBA for $4 \mathrm{~h}$ were subjected to H/R injury, as were H9c2 cells treated with Tg alone. (A and B) Western blot analysis of Beclin1, P62, LC3, ATF6, GRP78 and CHOP expression. Beclin1, P62, ATF6, GRP78, CHOP and LC3II/LC3I were normalized to GAPDH. (C) Fluorescence microscopy analysis of H9c2 cells transfected with mCherry-GFP-LC3. (D) Statistical analysis of fluorescent dots in H9c2 cells. Yellow spots indicate autophagosomes and red spots indicate autolysosomes in the merged images. Data are presented as the means \pm standard error of the mean of three independent experiments. "P<0.05 vs. Ctrl; ${ }^{\#} \mathrm{P}<0.05$ vs. H/R. 3-MA/3MA, 3-methyladenine; 4P/4-PBA, 4-phenylbutyrate; ATF6, activating transcription factor 6; CHOP, C/EBP homologous protein; Ctrl, Control; GFP, green fluorescent protein; GRP78, glucose-regulated protein 78; H/R, hypoxia/reoxygenation; LC3, microtubule-associated proteins 1A/1B light chain 3B; Rap, rapamycin; Tg, thapsigargin.

Effects of autophagy and ER stress on cell injury and apoptosis in H/R-treated H9c2 cells. CCK-8 and LDH assays were performed to analyze the extent of cell injury (Fig. 4A and B). It was revealed that cell injury was significantly decreased following pretreatment with Rap or 4-PBA compared with the H/R treatment group. In contrast, 3-MA pretreatment increased the injury of H/R-treated H9c2 cells. Subsequently, the apoptotic rate was measured by flow cytometry; pretreatment with Rap or 4-PBA significantly decreased the relative number of apoptotic H/R-treated
H9c2 cells, whereas 3-MA pretreatment significantly increased the number of apoptotic cells (Fig. 4C and D). Furthermore, BAX and caspase-3 levels were downregulated, whereas those of $\mathrm{Bcl} 2$ were markedly upregulated by the pretreatment of $\mathrm{H} / \mathrm{R}$-treated $\mathrm{H} 9 \mathrm{c} 2$ cells with Rap or 4-PBA; however, 3-MA pretreatment significantly increased the levels of BAX and caspase-3, but decreased those of $\mathrm{Bcl} 2$ in H/R-treated $\mathrm{H} 9 \mathrm{c} 2$ cells. In addition, cell injury and apoptosis were increased by Tg compared with the control group in H9c2 cells (Fig. 4E-I). 
A

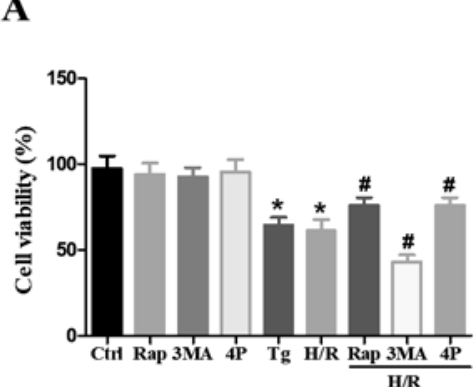

D

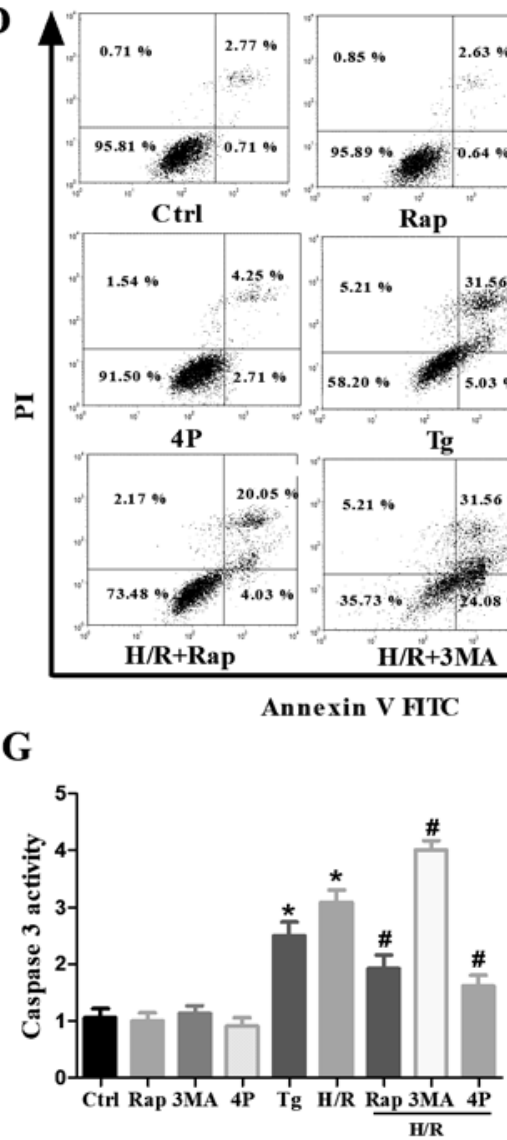

B

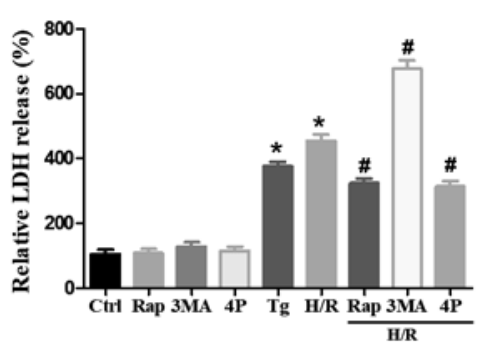

C

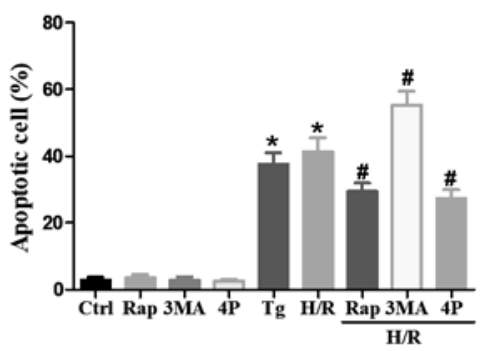

$\mathbf{E}$

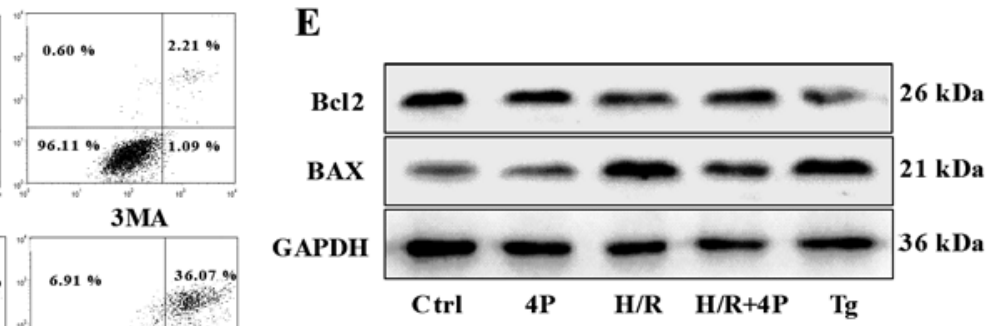

F

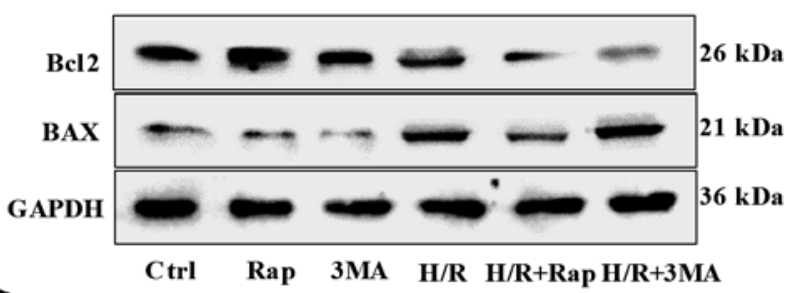

Ctrl Rap 3MA H/R H/R+RapH/R+3MA

H

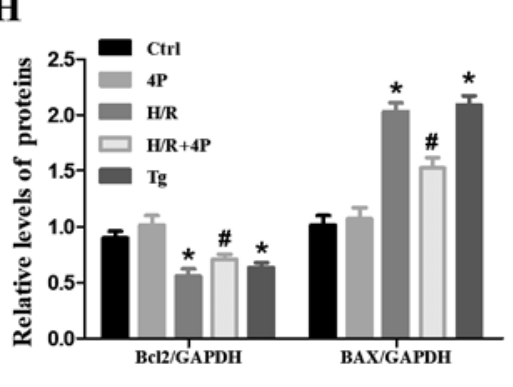

I

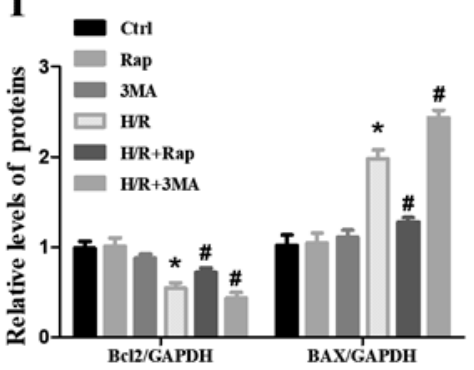

Figure 4. Effects of Rap, 3-MA, Tg and 4-PBA on the H/R-induced damage and apoptosis of H9c2 cells. Untreated H9c2 cells, or cells pretreated with Rap, 3-MA or 4-PBA for $4 \mathrm{~h}$ were subjected to H/R injury as were H9c2 cells treated with Tg alone. (A) Cell viability and (B) LDH release were determined via Cell Counting Kit-8 and LDH assays. (C and D) Apoptosis of H9c2 cells as determined via flow cytometry. (E and F) Western blot analysis of Bcl2 and BAX in H9c2 cells. (G) Caspase-3 activity as determined via a caspase-3 activity assay. (H and I) Semi-quantified expression of Bcl2 and BAX normalized to GAPDH. Data are presented as the means \pm standard error of the mean of three independent experiments. " $\mathrm{P}<0.05$ vs. Ctrl; ${ }^{~} \mathrm{P}<0.05$ vs. H/R. 3-MA/3MA, 3-methyladenine; 4P/4-PBA, 4-phenylbutyrate; Ctrl, Control; H/R, hypoxia/reoxygenation; PI, propidium iodide; Rap, Rapamycin; Tg, thapsigargin.

Effects of CHOP and Beclin1 on the interaction between autophagy and ER stress in H/R-treated $H 9 c 2$ cells. To further investigate the molecular mechanisms underlying the crosstalk between autophagy and ER stress, endogenous CHOP and Beclin1 levels were downregulated using siRNA. siRNA transfection significantly decreased the expression levels of CHOP and Beclin1 (Fig. S1). Western blot analysis revealed that CHOP siRNA significantly decreased the protein expression levels of CHOP in H/R-induced H9c2 cells. Notably, it also decreased Beclin1 levels and LC3-II/LC3-I ratio, and increased levels of P62 during H/R treatment (Fig. 5A). In addition, Beclin1 siRNA significantly decreased Beclin1 expression and LC3-II/LC3-I ratio, but increased P62 expression, in control and $\mathrm{H} / \mathrm{R}$-treated $\mathrm{H} 9 \mathrm{c} 2$ cells; it also upregulated the expression of ATF6, GRP78 and CHOP in H/R-induced H9c2 cells (Fig. 5B). The apoptotic rate was measured by flow cytometry, and it was demonstrated that CHOP siRNA decreased the number of apoptotic H/R-treated H9c2 cells, whereas Beclin1 siRNA transfection induced opposing effects (Fig. 5C and D). Additionally, CHOP siRNA decreased the levels of BAX, but increased those of Bcl2 in H/R-treated H9c2 cells, with Beclin1 siRNA increased the levels of BAX but decreased the levels of Bcl2 during H/R treatment as well as without H/R treatment (Fig. 5E). 

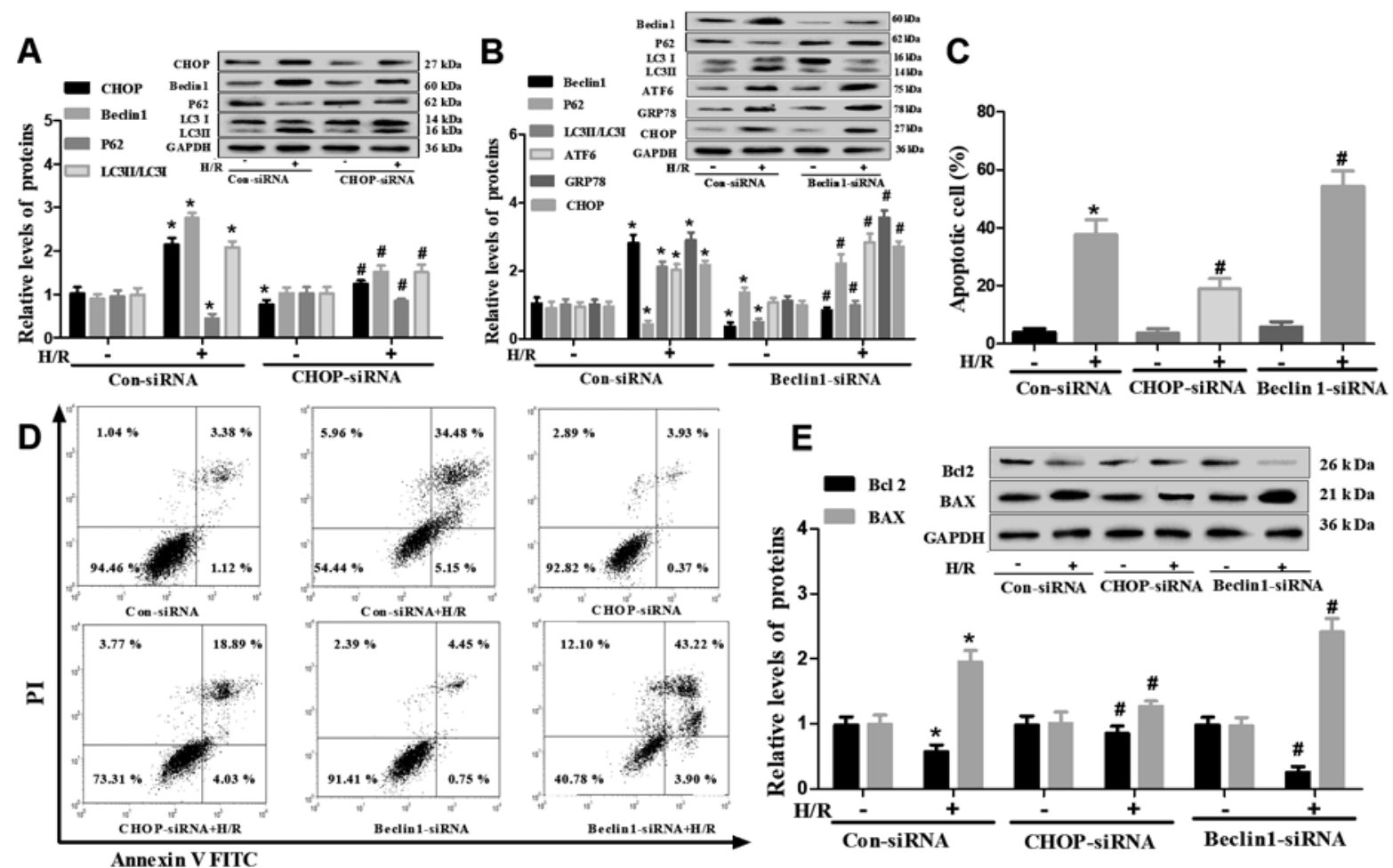

Figure 5. Effects of siRNA-mediated knockdown of Beclin1 and CHOP on the autophagy, endoplasmic reticulum stress and apoptosis of H/R-injured H9c2 cells. H9c2 cells were subjected to H/R injury following transfection with CHOP siRNA, Beclin1 siRNA or Con siRNA for $6 \mathrm{~h}$. (A and B) Western blot analysis of Beclin1, P62, LC3, ATF6, GRP78 and CHOP expression. Beclin1, P62, ATF6, GRP78, CHOP and LC3II/LC3I were normalized to GAPDH. (C and D) Apoptosis of H9c2 as determined via flow cytometry. (E) Western blot analysis of Bcl2 and BAX in H9c2 cells. Data are presented as the means \pm standard error of the mean of three independent experiments. ${ }^{*} \mathrm{P}<0.05$ vs. Con siRNA; ${ }^{*} \mathrm{P}<0.05$ vs. Con siRNA $+\mathrm{H} / \mathrm{R}$. ATF6, activating transcription factor 6; CHOP, C/EBP homologous protein; Con, Control; GRP78, glucose-regulated protein 78; H/R, hypoxia/reoxygenation; LC3, microtubule-associated proteins 1A/1B light chain 3B; PI, propidium iodide; siRNA, small interfering RNA.

\section{Discussion}

The present study investigated the effects of $H / R$ on the viability, damage and apoptosis of $\mathrm{H} 9 \mathrm{c} 2$ cells. Consistent with a previous study (15), the data revealed that $\mathrm{H} / \mathrm{R}$ decreased cell viability, increased cell damage and induced cell apoptosis in a time-dependent manner. Cell viability was reduced by $\sim 40 \%$ following a $12 / 6 \mathrm{~h}$ hypoxia/reperfusion period. Therefore, $12 / 6 \mathrm{~h}$ hypoxia/reperfusion treatment was selected to induce $\mathrm{H} / \mathrm{R}$ injury in subsequent experiments.

ER stress is an adaptive or protective response that decreases the accumulation of unfolded or misfolded proteins via the unfolded protein response (UPR) (7); however, under excessive stress, ER stress results in cell apoptosis (16). GRP78, a main indicator of ER stress, is a chaperone protein localized in the ER whose expression is typically increased in response to ER stress (17). ATF6 is activated by the accumulation of misfolded proteins and initiation of the UPR, inhibiting general protein translation (18). The UPR is a defense mechanism directed toward cellular adaptation to alleviate the unfolded protein load; however, prolonged stress is associated with the activation of proapoptotic proteins, such as CHOP (19). A previous study reported that ER stress is increased following $\mathrm{H} / \mathrm{R}$ treatment (15). Consistent with this study, the present findings indicated that GRP78, ATF6 and CHOP were upregulated in a time-dependent manner following $\mathrm{H} / \mathrm{R}$ injury.
Autophagy is an important intracellular bulk degradation process involving the lysosome-dependent turnover of damaged cytosolic proteins and organelles, which is critical for the maintenance of normal cell phenotypes and functions (20); however, the autophagic machinery may also contribute to cell death when pathological stress induces autophagy dysfunction (2). Beclin1 and LC3 are important proteins in autophagy. LC3 is a frequently used biomarker of the autophagosome membrane and reflects cellular autophagy activity. The P62 protein is an important substrate for autophagy degradation and has been defined as a marker of autophagic flux (7). Therefore, Beclin1, P62 and LC3 were selected as biomarkers of autophagy in the present study. Autophagy has been reported to be a regulatory target involved in H/R injury in cardiomyocytes (21). In the present study, it was observed that Beclin1 expression, LC3II lipidation and P62 degradation were increased in a time-dependent manner following $\mathrm{H} / \mathrm{R}$ injury. These results suggested that ER stress and autophagy were increased following H/R injury, consistent with the results of a previous study reporting that autophagy and ER stress are increased during myocardial $\mathrm{H} / \mathrm{R}$ injury (21).

Subsequently, the association between autophagy and ER stress was investigated in $\mathrm{H} 9 \mathrm{c} 2$ cells during $\mathrm{H} / \mathrm{R}$ injury. It was demonstrated that autophagy was decreased in H/R-treated H9c2 cells following 4-PBA treatment, whereas autophagy was increased following the treatment of $\mathrm{H} 9 \mathrm{c} 2$ cells with 
Tg. These results suggested that autophagy was attenuated following the inhibition of ER stress in H/R-injured H9c2 cells, and that increased ER stress following Tg treatment promoted autophagic flux. Therefore, these results indicated that autophagy was activated by ER stress during H/R injury, which is consistent with a previous study reporting that ER stress induces autophagy in mammalian cells $(22,23)$. A possible explanation for these results is that the UPR also leads to the transcriptional upregulation of a number of autophagy-associated genes required for the induction and construction of the autophagy machinery during ER stress (24).

To determine the effects of autophagy on ER stress during H/R-induced injury, ER stress was analyzed following the regulation of autophagy activity. The results indicated that promoting autophagy by pretreating H9c2 cells with Rap decreased ER stress during $\mathrm{H} / \mathrm{R}$ injury. In contrast, inhibiting autophagy by pretreating H9c2 cells with 3-MA increased ER stress during H/R injury. Therefore, the levels of ER stress during H/R injury were influenced by alterations in autophagy activity. This may be a result of the activation of autophagy relieving stress and reinstating homeostasis in the ER by removing unfolded proteins, protein aggregates and damaged organelles (25). Therefore, the present findings indicated the presence of a negative-feedback loop between autophagy and ER stress in $H 9 c 2$ cells during $H / R$ injury, with autophagy inhibiting ER stress but increased ER stress promoting autophagy.

Cardiomyocyte damage and apoptosis are prominent features of H/R injury. Therefore, the mechanisms of ER stress and autophagy that underlie cell damage and apoptosis in cardiomyocytes during $\mathrm{H} / \mathrm{R}$ injury were investigated. It was observed that cell damage and apoptosis were alleviated in $\mathrm{H} 9 \mathrm{c} 2$ cells during $\mathrm{H} / \mathrm{R}$ injury after inhibiting ER stress using 4-PBA. Conversely, promoting autophagy alleviated the apoptosis and damage of H/R-treated H9c2 cells, whereas inhibiting autophagy further promoted apoptosis and damage. These findings indicated that increasing ER stress increased apoptosis and damage, but that inducing autophagy served a protective role in $\mathrm{H} 9 \mathrm{c} 2$ cells during $\mathrm{H} / \mathrm{R}$ injury. Thus, combined with the conclusions that ER stress activated autophagy and increased apoptosis, the further activation of autophagy alleviated ER stress and apoptosis in H9c2 cells during H/R injury. Therefore, it was hypothesized that apoptosis is induced depending on the levels of ER stress, and that autophagy serves a protective role by alleviating ER stress in $\mathrm{H} 9 \mathrm{c} 2$ cells during H/R injury. These findings are consistent with previous studies that reported that ER stress serves an important role in the H/R-induced apoptosis of cardiomyocytes $(15,26)$. In addition, these results supported the previous finding that increased autophagy alleviates H/R injury (11). Conversely, it has been reported elsewhere that inhibition of autophagy alleviates H/R injury (27). One possible explanation for this discrepancy may be that the distinct effects of autophagy are dependent on the duration or method of $H / R$ treatment.

To further determine the effects of $\mathrm{H} / \mathrm{R}$ injury on cell apoptosis, the activity (caspase-3) or expression (BAX and $\mathrm{Bcl} 2$ ) of apoptosis-associated proteins was investigated. Caspase-3 is processed into cleaved-caspase- 3 during the early stages of apoptosis, and the expression of caspase- 3 is positively associated with the rate of apoptosis in cells (28). $\mathrm{Bcl} 2$ is an important protein in promoting cellular survival and inhibiting the actions of proapoptotic proteins (29). Conversely, BAX, a member of the Bcl 2 family, induces proapoptotic effects (30). The present results indicated that 4-PBA and Rap pretreatment decreased the activity of caspase- 3 and expression of BAX, but increased the levels of $\mathrm{Bcl} 2$ in $\mathrm{H} 9 \mathrm{c} 2$ cells during $\mathrm{H} / \mathrm{R}$ injury; however, 3-MA pretreatment induced opposing effects. These findings further supported the data from the flow cytometry assays.

$\mathrm{CHOP}$ is a major point of convergence for the three major upstream ER stress transducers, and is also the most extensively characterized factor in the transition from ER stress to apoptosis $(31,32)$. Beclin1 is required for the initiation of autophagy (33). To investigate the molecular mechanisms underlying the roles of ER stress and autophagy in the apoptosis of H/R-induced H9c2 cells, the levels of CHOP and Beclin1 were downregulated with siRNA. It was observed that CHOP siRNA decreased autophagy and attenuated the apoptosis of $\mathrm{H} 9 \mathrm{c} 2$ cells during $\mathrm{H} / \mathrm{R}$ injury, indicating that the activation of autophagy and the promotion of apoptosis depended on the increased expression of CHOP in $\mathrm{H} / \mathrm{R}$ injury. Conversely, Beclin1 siRNA inhibited autophagy, but aggravated ER stress and cell apoptosis. These results further indicated that autophagy serves a protective role against ER stress and apoptosis in H9c2 cells during H/R injury.

In summary, the results of the present study provided further evidence that H/R induces apoptosis and autophagy via ER stress in cardiomyocytes. The findings suggested that CHOP serves an important role in ER stress-induced autophagy and apoptosis. Additionally, autophagy, as an adaptive response, alleviated ER stress and ER stress-induced apoptosis in $\mathrm{H} 9 \mathrm{c} 2$ cells during $\mathrm{H} / \mathrm{R}$ injury. The novel findings that ER stress-induced autophagy serves a protective role in H9c2 cells during H/R injury may aid future research.

\section{Acknowledgements}

Not applicable.

\section{Funding}

The present study was funded by the National Natural Science Foundation of China (grant no. 81660152) and the Natural Science Foundation of Jiangxi Province (grant no. 20181BAB205004).

\section{Availability of data and materials}

The datasets used and/or analyzed during the present study are available from the corresponding author on reasonable request.

\section{Authors' contributions}

LY and XG were involved in the conception and design of the experiments. LY supervised the work. GPG, WYH, PHZ and JZ performed the experiments. GPG, HY and SYL analyzed data. GPG contributed to drafting and revising the manuscript. $\mathrm{XG}$ approved the final version to be published. 


\section{Ethics approval and consent to participate}

Not applicable.

\section{Patient consent for publication}

Not applicable.

\section{Competing interests}

The authors declare that they have no competing interests.

\section{References}

1. Kalkman DN, Aquino M, Claessen BE, Baber U, Guedeney P, Sorrentino S, Vogel B, de Winter RJ, Sweeny J, Kovacic JC, et al: Residual inflammatory risk and the impact on clinical outcomes in patients after percutaneous coronary interventions. Eur Heart J 39: 4101-4108, 2018.

2. Ma X, Liu H, Foyil SR, Godar RJ, Weinheimer CJ, Hill JA and Diwan A: Impaired autophagosome clearance contributes to cardiomyocyte death in ischemia/reperfusion injury. Circulation 125: 3170-3181, 2012.

3. Yang Y, Ding S, Xu G, Chen F and Ding F: MicroRNA-15a inhibition protects against hypoxia/reoxygenation-induced apoptosis of cardiomyocytes by targeting mothers against decapentaplegic homolog 7. Mol Med Rep 15: 3699-3705, 2017.

4. Liao F, Zheng Y, Cai J, Fan J, Wang J, Yang J, Cui Q, Xu G, Tang C and Geng B: Catestatin attenuates endoplasmic reticulum induced cell apoptosis by activation type 2 muscarinic acetylcholine receptor in cardiac ischemia/reperfusion. Sci Rep 5: 16590, 2015

5. Yao T, Ying X, Zhao Y, Yuan A, He Q, Tong H, Ding S, Liu J, Peng X, Gao E, et al: Vitamin D receptor activation protects against myocardial reperfusion injury through inhibition of apoptosis and modulation of autophagy. Antioxid Redox Signal 22: 633-650, 2015

6. Ma Y, Shimizu Y, Mann MJ, Jin Y and Hendershot LM: Plasma cell differentiation initiates a limited ER stress response by specifically suppressing the PERK-dependent branch of the unfolded protein response. Cell Stress Chaperones 15: 281-293, 2010.

7. Rashid HO, Yadav RK, Kim HR and Chae HJ: ER stress: Autophagy induction, inhibition and selection. Autophagy 11: 1956-1977, 2015

8. Gatica D, Chiong M, Lavandero S and Klionsky DJ: Molecular mechanisms of autophagy in the cardiovascular system. Circ Res 116: 456-467, 2015

9. Salabei JK and Conklin DJ: Cardiovascular autophagy: Crossroads of pathology, pharmacology and toxicology. Cardiovasc Toxicol 13: 220-229, 2013.

10. Guo JJ, Xu FQ, Li YH, Li J, Liu X, Wang XF, Hu LG and An Y: Alginate oligosaccharide alleviates myocardial reperfusion injury by inhibiting nitrative and oxidative stress and endoplasmic reticulum stress-mediated apoptosis. Drug Des Dev Ther 11: 2387-2397, 2017.

11. Li H, Zhang X, Tan J, Sun L, Xu LH, Jiang YG, Lou JS, Shi XY and Mi WD: Propofol postconditioning protects $\mathrm{H} 9 \mathrm{c} 2$ cells from hypoxia/reoxygenation injury by inducing autophagy via the SAPK/JNK pathway. Mol Med Rep 17: 4573-4580, 2018.

12. Ding WX, Ni HM, Gao W, Hou YF, Melan MA, Chen X, Stolz DB, Shao ZM and Yin XM: Differential effects of endoplasmic reticulum stress-induced autophagy on cell survival. J Biol Chem 282: 4702-4710, 2007.

13. Wang C, Zhang S, Ma R, Zhang X, Zhang C, Li B, Niu Q, Chen J, $\mathrm{Xia}$ T, Li P, et al: Roles of endoplasmic reticulum stress, apoptosis and autophagy in 2,2',4,4'-tetrabromodiphenyl ether-induced rat ovarian injury. Reprod Toxicol 65: 187-193, 2016.

14. Livak KJ and Schmittgen TD: Analysis of relative gene expression data using real-time quantitative PCR and the 2(-Delta Delta C(T)) method. Methods 25: 402-408, 2001.

15. Du Y, Wang M, Liu X, Zhang J, Xu X, Xu H, Sun G and Sun X: Araloside C prevents hypoxia/reoxygenation-induced endoplasmic reticulum stress via increasing heat shock protein 90 in H9c2 cardiomyocytes. Front Pharmacol 9: 180, 2018.
16. Sano R and Reed JC: ER stress-induced cell death mechanisms Biochim Biophys Acta 1833: 3460-3470, 2013.

17. Lee AS: The ER chaperone and signaling regulator GRP78/BiP as a monitor of endoplasmic reticulum stress. Methods 35: 373-381, 2005.

18. Harding HP, Novoa I, Zhang Y, Zeng H, Wek R, Schapira M and Ron D: Regulated translation initiation controls stress-induced gene expression in mammalian cells. Mol Cell 6: 1099-1108, 2000 .

19. Gorman AM, Healy SJ, Jäger R and Samali A: Stress management at the ER: Regulators of ER stress-induced apoptosis. Pharmacol Ther 134: 306-316, 2012.

20. Przyklenk K, Dong Y, Undyala VV and Whittaker P: Autophagy as a therapeutic target for ischaemia/reperfusion injury? Concepts, controversies, and challenges. Cardiovasc Res 94: 197-205, 2012.

21. Matsui Y, Takagi H, Qu X, Abdellatif M, Sakoda H, Asano T, Levine B and Sadoshima J: Distinct roles of autophagy in the heart during ischemia and reperfusion: Roles of AMP-activated protein kinase and Beclin 1 in mediating autophagy. Circ Res 100: 914-922, 2007.

22. Høyer-Hansen M and Jäättelä M: Connecting endoplasmic reticulum stress to autophagy by unfolded protein response and calcium. Cell Death Differ 14: 1576-1582, 2007.

23. Kawakami T, Inagi R, Takano H, Sato S, Ingelfinger JR, Fujita $T$ and Nangaku M: Endoplasmic reticulum stress induces autophagy in renal proximal tubular cells. Nephrol Dial Transplant 24: 2665-2672, 2009.

24. Rouschop KM, van den Beucken T, Dubois L, Niessen H, Bussink J, Savelkouls K, Keulers T, Mujcic H, Landuyt W, Voncken JW, et al: The unfolded protein response protects human tumor cells during hypoxia through regulation of the autophagy genes MAP1LC3B and ATG5. J Clin Invest 120: 127-141, 2010.

25. Doyle KM, Kennedy D, Gorman AM, Gupta S, Healy SJ and Samali A: Unfolded proteins and endoplasmic reticulum stress in neurodegenerative disorders. J Cell Mol Med 15: 2025-2039, 2011.

26. Gao Y, Yang H, Chi J, Xu Q, Zhao L, Yang W, Liu W and Yang W: Hydrogen gas attenuates myocardial ischemia reperfusion injury independent of postconditioning in rats by attenuating endoplasmic reticulum stress-induced Autophagy. Cell Physiol Biochem 43: 1503-1514, 2017.

27. Wang Y, Wang Q, Zhang L, Ke Z, Zhao Y, Wang D, Chen H, Jiang X, Gu M, Fan S and Huang C: Coptisine protects cardiomyocyte against hypoxia/reoxygenation-induced damage via inhibition of autophagy. Biochem Biophys Res Commun 490: 231-238, 2017.

28. Mirzayans R, Andrais B, Kumar P and Murray D: The growing complexity of cancer cell response to DNA-damaging agents: Caspase 3 mediates cell death or survival? Int J Mol Sci 17: pii: E708, 2016.

29. Hardwick JM and Soane L: Multiple functions of BCL-2 family proteins. Cold Spring Harb Perspect Biol 5: pii: a008722, 2013.

30. Sobhan PK, Seervi M, Deb L, Varghese S, Soman A, Joseph J, Mathew KA, Raghu G, Thomas G, E S, et al: Calpain and reactive oxygen species targets Bax for mitochondrial permeabilisation and caspase activation in zerumbone induced apoptosis. PLoS One 8: e59350, 2013.

31. Su J, Zhou L, Kong X, Yang X, Xiang X, Zhang Y, Li X and Sun L: Endoplasmic reticulum is at the crossroads of autophagy, inflammation, and apoptosis signaling pathways and participates in the pathogenesis of diabetes mellitus. J Diabetes Res 2013: 193461, 2013

32. Marciniak SJ, Yun CY, Oyadomari S, Novoa I, Zhang Y, Jungreis R, Nagata K, Harding HP and Ron D: CHOP induces death by promoting protein synthesis and oxidation in the stressed endoplasmic reticulum. Genes Dev 18: 3066-3077, 2004.

33. Kang R, Zeh HJ, Lotze MT and Tang D: The beclin 1 network regulates autophagy and apoptosis. Cell Death Differ 18: 571-580, 2011.

This work is licensed under a Creative Commons Attribution-NonCommercial-NoDerivatives 4.0 International (CC BY-NC-ND 4.0) License. 